\title{
Revealing the Kinetic Advantage of a Competitive Small- Molecule Immunoassay by Direct Detection
}

\author{
Renee J. Tran, Krystal L. Sly, John C. Conboy* \\ Department of Chemistry, University of Utah, 315 South 1400 East RM. 2020, Salt Lake City, \\ Utah 84112 \\ *conboy@chem.utah.edu
}

\section{TABLE OF CONTENTS}

\section{Supporting Figures}

Figure S1. Equilibrium adsorption isotherms of morphine (green), methadone (blue), and cocaine (red) to (A) anti-morphine, (B) anti-methadone, and (C) anti-cocaine. Solid lines are fits to Equation 2. Error bars represent the standard deviation of triplicate measurements on separate capture surfaces.

Figure S2. Computed ßaverage values as a function of wavelength for morphine (green), methadone (blue), and cocaine (red).

\section{Supporting Table}

Table S1. Equilibrium binding affinity (Keq) values of morphine, methadone, and cocaine binding to anti-morphine, anti-methadone, and anti-cocaine.

\section{Supporting Experimental Section}

3.1 Background Subtraction and Normalization of the SHG Signal

3.2 SHG Equilibrium Binding Assays.

3.3 Wavelength Dependent Nonlinear Susceptibilities 


\section{Supporting Figures}

Figure S1. Equilibrium adsorption isotherms of morphine (green), methadone (blue), and cocaine (red) to (A) anti-morphine, (B) anti-methadone, and (C) anti-cocaine. Solid lines are fits to Equation 2. Error bars represent the standard deviation of triplicate measurements on separate capture surfaces.

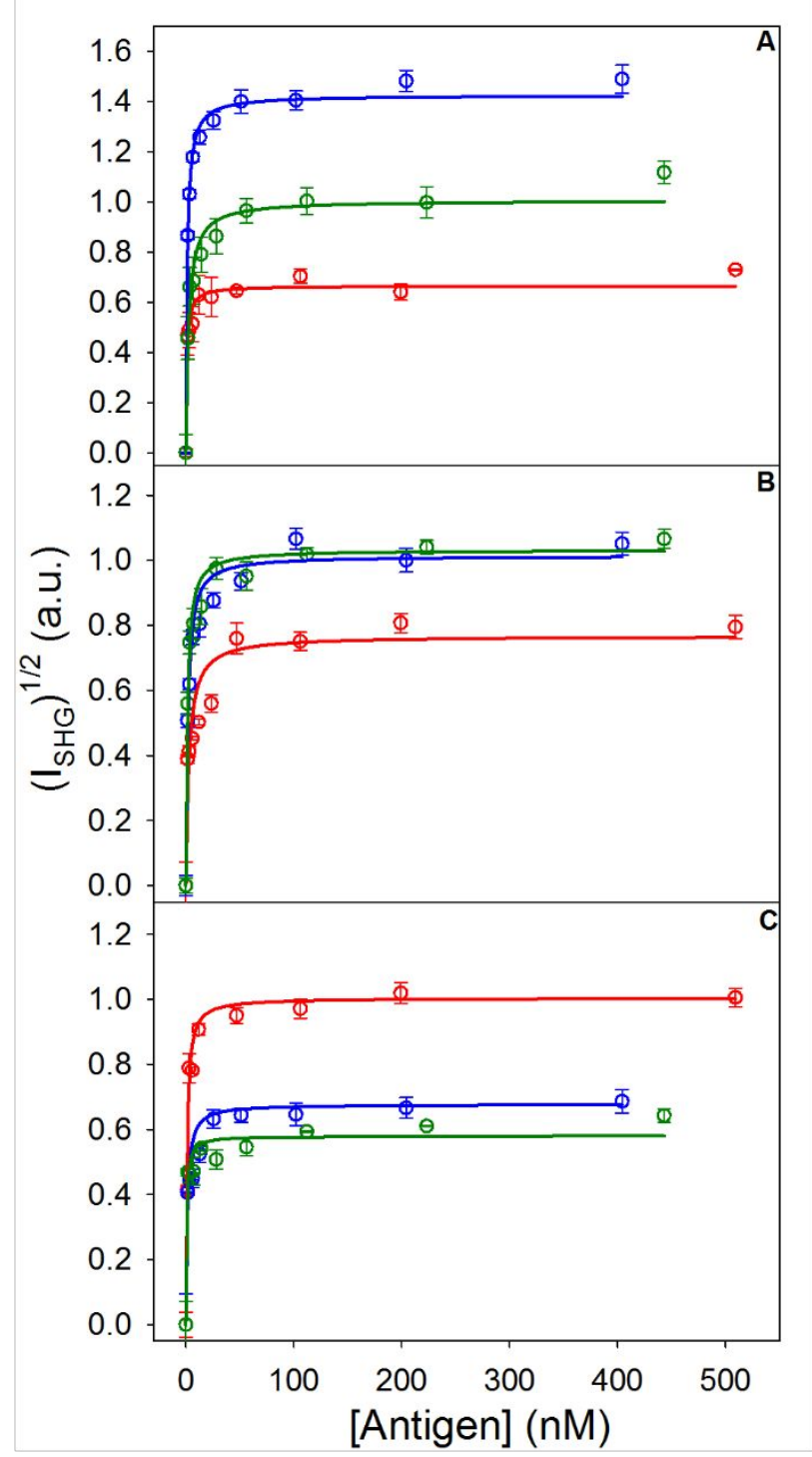


Figure S2. Computed $\beta_{\text {average }}$ values as a function of wavelength for morphine (green), methadone (blue), and cocaine (red).

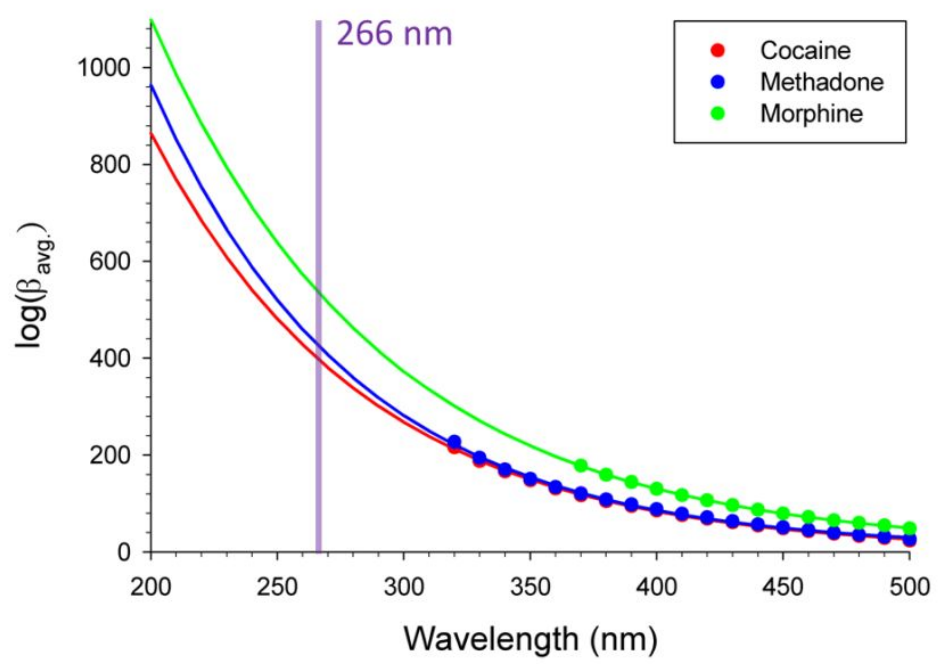




\section{Supporting Table}

Table S1. Equilibrium binding affinity $\left(\mathrm{K}_{\mathrm{eq}}\right)$ values of morphine, methadone, and cocaine binding to anti-morphine, anti-methadone, and anti-cocaine.

\begin{tabular}{c|c|}
\multicolumn{1}{c}{} & $\begin{array}{c}\mathbf{K}_{\mathbf{e q}} \\
\times \mathbf{1 0}^{\mathbf{9}} \mathbf{M}^{-\mathbf{1}}\end{array}$ \\
\hline Anti-morphine & \\
\hline Morphine & $0.4 \pm 0.1$ \\
Methadone & $1.9 \pm 0.2$ \\
Cocaine & $2.5 \pm 0.6$ \\
Morphine-HRP & $0.7 \pm 0.1$ \\
\hline Anti-methadone & \\
\hline Morphine & $1.5 \pm 0.2$ \\
Methadone & $1.3 \pm 0.2$ \\
Cocaine & $0.4 \pm 0.1$ \\
\hline Anti-cocaine & \\
\hline Morphine & $3.3 \pm 1.3$ \\
Methadone & $1.7 \pm 0.4$ \\
Cocaine & $2.1 \pm 0.5$
\end{tabular}




\section{Supporting Experimental Section}

\subsection{Background Subtraction and Normalization of the SHG Signal}

In order to obtain accurate and statistically meaningful results from the SHG immunoassay, a method was needed to directly compare SHG signals recorded from different experiments. In order to account for the background signal from the sensor surface due to the immobilized antibodies and offsets in the detector electronics, and/or contributions due to scattered excitation light and multi-photon fluorescence events, a PBS buffer at $\mathrm{pH} 7.5$ was introduced into the sample cell at the beginning of each assay. The signal measured from PBS pH 7.5 was then used to account for any shift in the SHG signal's offset and subtract the SHG background from the sensor prior to analyte adsorption. In order to compare results from different assays, the SHG data were normalized by measuring the SHG intensity from a 10 $\mathrm{mM} \mathrm{KOH}$ solution which was rinsed through the cell after the completion of an experiment. This solution removes the antibody capture surface resulting in a bare silica interface, provided a clean and constant composition of the surface as a reference. The use of this single point normalization effectively corrects for differences in collection efficiency, alignment of the lasers and detector sensitivity from experiment to experiment. The SHG signal was corrected for background and normalized using the Equation S1:

$$
\text { normalized } I^{S H G}=\frac{I^{S H G}-I_{P B S}^{S H G}}{I_{K O H}^{S H G}-I_{P B S}^{S H G}}
$$

where $I^{S H G}$ is the measured SHG intensity, and $I_{K O H}^{S H G}$ and $I_{P B S}^{S H G}$ are the intensities collected from $10 \mathrm{mM}$ $\mathrm{KOH}$ and $\mathrm{PBS} \mathrm{pH} 7.5$ respectively.

\subsection{SHG Equilibrium Binding Assays.}

The rates of adsorption of morphine, methadone, and cocaine to the antibodies can be calculated using the measured rates of dissociation listed in Table 2 and the equilibrium binding affinities of each drug-antibody pair. Equilibrium adsorption isotherms of the drugs to antimorphine, anti-methadone, and anti-cocaine antibodies at bulk concentrations ranging from 1.5 to $509 \mathrm{nM}$ were measured by SHG, shown in Figure S1. The SHG data was fit to Equation 2 to obtain the equilibrium binding affinities $\left(\mathrm{K}_{\mathrm{eq}}\right)$ listed in Table $\mathrm{S} 1$, which were used to calculate the rates of adsorption in Table 2 using

$$
K_{e q}=\frac{k_{o n}}{k_{o f f}}
$$

where $\mathrm{k}_{\text {off }}$ is the measured rate of desorption. Although it appears that the antibodies are not selective at equilibrium, the adsorption of the non-target drugs is reduced after washing as shown by the measured SHG intensities for the non-target drugs in Figure 3, which indicates the wash step is essential to obtain selectivity in a direct immunoassay in the absence of competition.

\subsection{Wavelength Dependent Nonlinear Susceptibilities}

The theoretically calculated second order nonlinear susceptibilities ( $\left.\beta_{\text {average }}\right)$ have been computed for morphine, methadone and cocaine as a function of wavelength using MOPAC (Molecular Orbital PACkage, MOPAC2016, James J. P. Stewart, Stewart Computational Chemistry, Colorado Springs, CO, USA, HTTP://OpenMOPAC.net (2016).) and are shown in Figure S2. Due to the large $\beta_{\text {average }}$ values below $320 \mathrm{~nm}$ for cocaine and methadone and below $370 \mathrm{~nm}$ for morphine, MOPAC was not able to compute these values due to the floating point limit of the program, so the values were extrapolated assuming a Lorentzian line shape for the 
resonance, which was approximated by an exponential decay on the low energy (long wavelength) side of the resonance, shown in Figure S2 as the solid line. The computed spectra show a significant increase in $\beta_{\text {average }}$ at wavelengths below $400 \mathrm{~nm}$, and a significant in enhancement is computed at $266 \mathrm{~nm}$ for morphine, methadone and cocaine. 